\title{
Diferencias entre niños y adultos por el nuevo coronavirus 2019, SARS-CoV-2/COVID-19
}

\author{
Differences between children and adults by the new \\ coronavirus 2019, SARS-CoV-2 (COVID-19) \\ Iván Benavides Reina,* Eduardo López Medina, Pio López López ${ }^{\S}$ \\ * Fellow en Infectología Pediátrica. \\ ₹ Infectólogo Pediatra, Epidemiólogo. Centro Médico Imbanaco, Centro de Estudios en Infectología Pediátrica (CEIP). \\ $\S$ Infectólogo Pediatra. CEIP. Presidente de la Sociedad Latinoamericana de Infectología Pediátrica (SLIPE). \\ Universidad del Valle. Cali, Colombia.
}

\section{RESUMEN}

En diciembre de 2019 desde China se reportaron casos que fueron llamados inicialmente «neumonía de origen desconocido»; el agente causal de esta neumonía es un nuevo coronavirus, el cual fue nombrado coronavirus 2 del síndrome respiratorio agudo severo (SARS-CoV-2). EI término COVID-19 se utiliza para definir la enfermedad clínica causada por SARS-CoV-2. En niños la enfermedad parece ser más leve que en los adultos, por lo que se han propuesto varias hipótesis para explicar este comportamiento. Sin embargo, los lactantes, niños y adolescentes con comorbilidades tienen cuadros relativamente más severos. En esta revisión presentamos las diferencias entre niños y adultos con COVID-19, las manifestaciones clínicas (enfatizando en el síndrome postinflamatorio multisistémico), las pruebas diagnósticas y el papel de los niños en la transmisión comunitaria de la infección.

Palabras clave: Coronavirus, SARS, COVID-19, SARS-CoV-2, 2019-nCoV, niños, SIMS-TAC.

\section{DIFERENCIAS ENTRE NIÑOS Y ADULTOS CON COVID-19}

El Centro para el Control y Prevención de Enfermedades de China reportó que de los 72,314 casos de COVID-19, sólo $2 \%$ se presentó en menores de 19 años, $1 \%$ en menores de 10 años y no se informaron muertes. La realidad en mayores de 19 años es distinta con una mortalidad de $2.3 \%$ siendo mayor en pacientes entre 70 años.

Financiamiento: Ninguno.

Conflicto de intereses: Ninguno.

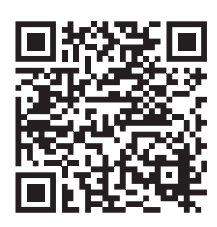

ABSTRACT

In December 2019, cases that were initially called «pneumonia of unknown origin" were reported in China. The causative agent of this pneumonia is a new coronavirus, named coronavirus 2 of severe acute respiratory syndrome (SARS-CoV-2). The term COVID-19 is used to define the disease caused by SARSCoV-2. In children the disease appears to be milder than in adults, and several hypotheses have been proposed to explain this presentation. However, infants, adolescents, and children with comorbidities have more severe conditions. In this review, we present the differences between children and adults with COVID-19; their clinical manifestations emphasizing postinflammatory multi-system syndrome, diagnostic tests and the role of children in community transmission of infection.

Keywords: Coronavirus, SARS, COVID-19, SARS-CoV-2, 2019nCoV, children, MIS-C.

Otro reporte de casos retrospectivo en China (exclusivamente de población pediátrica) incluyó 2,146 pacientes, $90 \%$ presentaron síntomas respiratorios leves y moderados o fueron asintomáticos. La proporción de casos graves y críticos fue de $10.6 \%$ en menores de un año, $7.3 \%$ entre $1-5$ años, $4.2 \%$ entre 6-10 años, $4.1 \%$ entre $11-15$ años y $3.0 \%$ para el grupo de edad de $\geq 16$ años. Sin embargo, la mayoría de los casos con síntomas graves pertenecían al grupo de sospecha clínica de COVID-19 (no tenían prueba de laboratorio confirmatoria). Queda por determinar si estos casos graves y críticos en el

Citar como: Benavides RI, López ME, López LP. Diferencias entre niños y adultos por el nuevo coronavirus 2019, SARS-CoV-2/COVID-19. Rev Latin Infect Pediatr. 2020; 33 (4): 165-173.https://dx.doi.org/10.35366/96845 
grupo sospechoso fueron por COVID-19 o infecciones por otros patógenos (por ejemplo el virus sincitial respiratorio [VSR]). Sólo se reportó una muerte en un adolescente de 14 años. La mayoría de los casos confirmados fueron secundarios a su exposición a contactos familiares y se recuperaron de una a dos semanas después del inicio de los síntomas. ${ }^{2}$

En Italia, el reporte de 22,512 casos publicado en JAMA, sólo 1.2\% correspondía a menores de 18 años y no se reportaron muertes.

En tiempos en los que recibimos abundante información sobre COVID-19, es importante recordar que los menores de cinco años especialmente, presentan a menudo infecciones respiratorias y éstas se incrementan con la llegada del invierno. En un estudio retrospectivo al comienzo del brote en Wuhan, se incluyeron 366 niños con infecciones respiratorias. Los patógenos detectados con mayor frecuencia fueron el virus de la influenza $A$ (en 23 pacientes) y el virus de la influenza $B$ (en 20 pacientes) y el SARS-CoV-2 se detectó en seis pacientes (1.6\%). ${ }^{3}$

Por otro lado, se ha visto que la eliminación del virus en las secreciones respiratorias y en las heces es más prolongada en los niños con síntomas leves que en los adultos, hecho que ocasiona un gran desafío para el control de la infección. ${ }^{1}$

Hay escasa evidencia de la transmisión vertical en mujeres embarazadas y COVID-19 en el tercer trimestre de embarazo. Está por demostrar si la infección perinatal puede tener efectos adversos sobre recién nacidos. El riesgo de infectar a un recién nacido por gotas o por contacto es igual que en la población general. ${ }^{3}$

La presencia de comorbilidades médicas como hipertensión, diabetes, enfermedad pulmonar crónica y enfermedad cardiovascular son factores de riesgo de enfermedad grave en adultos, y la relativa falta de comorbilidades en los niños puede contribuir a la disparidad en la gravedad de COVID-19 entre los grupos de edad. Dada la baja prevalencia de enfermedad grave y crítica en niños, es difícil determinar la contribución de las comorbilidades preexistentes a la gravedad de COVID-19.

Posiblemente las comorbilidades en pediatría como displasia broncopulmonar, enfermedad cardiaca congénita, asma, pueden dar lugar a un aumento en el riesgo de COVID-19 grave.

Hay pocos informes de enfermedad grave en pacientes inmunocomprometidos con COVID-19 a pesar de ser receptores de agentes inmunosupresores y quimioterapias. La mayoría tienen un curso leve que podría aludir al papel sustancial que el sistema inmunitario del huésped juega en el desarrollo de enfermedades graves. ${ }^{4}$

\section{¿POR QUÉ LA ENFERMEDAD EN NIÑOS POR COVID-19 ES MENOS SEVERA?}

Se desconoce por qué la mayoría de los casos de COVID-19 de los niños fueron menos graves que en los adultos. Esto puede estar relacionado con una menor expresión de los factores del huésped requeridos para la replicación viral y las diferencias en la magnitud de las respuestas inmunes innatas y adaptativas. Los altos niveles de citocinas (tormenta de citocinas) se han asociado con una mayor carga viral, pérdida de la función pulmonar, lesión pulmonar y un desenlace fatal.

Las tasas del síndrome de dificultad respiratoria aguda (SDRA) son más bajas en los niños y aumentan con la edad, lo que sugiere un papel para factores protectores del huésped en los pulmones de los niños, por ejemplo, las bajas concentraciones de la enzima convertidora de angiotensina II (ECA-II), conocida como receptor celular del SARS-CoV. El SARS-CoV-2 tiene cierta homología de aminoácidos con el SARS-CoV y puede usar ECA-II como receptor y la proteasa celular transmembrana de serina 2 (TMPRSS2) para activar la proteína viral Spike (S) implicada en la fusión de membranas.

ECA-II modula la vasoconstricción para mantener homeostasis y se expresa en la mucosa oral, nasal, el tracto respiratorio y el intestino, lo que explica en cierta parte los síntomas a nivel olfatorio, respiratorio y gastrointestinal. El factor nuclear NF-kB implicado en enfermedades inflamatorias también se encuentra en menor concentración en niños y debe evaluarse como un factor protector del huésped en el SARSCoV-2 pediátrico versus adulto, incluso fuera del contexto de las infecciones por SARS-CoV-2. Los análisis de biopsias pulmonares de adultos de casos de SARS-CoV-1 sugieren que la infección por SARS-CoV-2 más leve en niños puede ser impulsado por niveles intrínsecamente más bajos de citocinas inflamatorias (por ejemplo menores concentraciones de IL-6), una mayor proporción de linfocitos respecto a neutrófilos en sangre y menor infiltración monocítica que los adultos. Por lo tanto, el estudio del papel de los neutrófilos y macrófagos en las infecciones por SARS-CoV-2 debe seguir siendo evaluado y compararse entre niños y adultos. El aumento observado en la patología pulmonar en lactantes menores de 
Rev Latin Infect Pediatr 2020; 33 (4): 165-173

un año puede deberse a niveles inapropiados de actividad de las células T CD8+, que juegan un papel importante en la eliminación viral, y esta inmadurez del sistema inmune puede ser explicado por el efecto de la inmunidad fetal tolerogénica. ${ }^{4}$

Las nuevas preocupaciones en niños es la descripción de un síndrome inflamatorio multisistémico asociado temporalmente a SARS-CoV-2 (PIMS-TS) que podría representar un síndrome inflamatorio postinfeccioso mediado por anticuerpos o inmunocomplejos. La comprensión de este fenómeno inflamatorio podría proporcionar información vital sobre las respuestas inmunes al SARS-CoV-2 y podría tener relevancia tanto para adultos como para niños. ${ }^{5}$

Aunque la vacuna BCG se ha propuesto para la protección contra COVID-19, recientes estudios no respaldan la idea de que esta vacuna aplicada en la infancia tenga un efecto protector en la edad adulta. ${ }^{6}$

Otra explicación propuesta para el curso leve de la enfermedad en niños es la exposición frecuente a esta edad de infecciones respiratorias (por ejemplo VSR o HCoV) y vacunación. Se cree que estos efectos podrían estar mediados en parte por los efectos heterólogos sobre la inmunidad adaptativa, como la reactividad cruzada mediada por células T y el número de anticuerpos neutralizantes, pero también por la potenciación de la respuesta inmune innata. $^{7}$

\section{MANIFESTACIONES CLÍNICAS}

Los síntomas más comunes de COVID-19 en pediatría incluyen fiebre, tos, rinorrea, vómito, diarrea y mialgias, además hay un menor porcentaje de dificultad respiratoria si se compara con los adultos.

En la mayoría de los reportes pediátricos informan que aproximadamente $50 \%$ de los niños sintomáticos cursan con fiebre y $54 \%$ con tos, mientras que la fiebre y la tos se identificaron en 71 y $80 \%$ de los adultos, respectivamente, y faringitis en $30 \%$ de los casos. ${ }^{4}$ Es decir, la ausencia de fiebre no descarta COVID-19.

Las estimaciones precisas de enfermedad grave y muerte debido a COVID-19 entre los niños no son precisas debido a la ausencia de verdaderos denominadores de la población. ${ }^{5}$ Los niños menores de cinco años tienen mayores porcentajes de hospitalización y severidad, especialmente los menores de un año, los cuales tienen tasas de hospitalización de $90 \%$. El uso de ventilación mecánica es más frecuente en recién nacidos pretérmino e infantes con enfermedades congénitas respiratorias, cardiacas y neurológicas. ${ }^{8,9}$

\section{SIINDROME INFLAMATORIO MULTISISTÉMICO EN NIÑOS Y ADOLESCENTES}

En Bérgamo, Italia, la región más afectada por COVID-19, la incidencia mensual de la enfermedad de Kawasaki aumentó 30 veces a la observada en cinco años. Esta enfermedad es una vasculitis pediátrica aguda rara, con aneurismas en la arteria coronaria como su principal complicación; el diagnóstico se basa en la presencia de fiebre persistente mayor de cinco días que puede estar acompañado con varias manifestaciones clínicas (exantema, linfadenopatía en cuello, inyección conjuntival no supurativa bilateral, cambios en las mucosas y extremidades, lengua en fresa, labios fisurados, edema de manos y pies), asociado en la mayoría de las ocasiones con síntomas gastrointestinales como dolor abdominal, vómito y diarrea.

Se han reportado casos de Kawasaki completo (presencia de fiebre y más de cuatro signos clínicos) e incompleto (fiebre y dos o tres signos clínicos); además estos casos se presentaron en niños de mayor edad (nueve a 17 años) al contrario que el Kawasaki clásico. ${ }^{5}$

En el Reino Unido y América del Norte los pediatras han identificado a un pequeño grupo de niños que se presentan con choque y que han requerido uso de inotrópicos, algunos de los cuales tienen aneurismas en las arterias coronarias, y otro grupo de niños con enfermedades menos graves similares a Kawasaki.

Las hipótesis iniciales son que este síndrome puede estar relacionado con COVID-19 de acuerdo con las pruebas de laboratorio iniciales. Los niños han sido tratados con antiinflamatorios, que incluyen inmunoglobulina parenteral y esteroides.

La OMS enfatiza la necesidad de caracterizar este síndrome y sus factores de riesgo, comprender la causalidad y describir las intervenciones de tratamiento. Se ha desarrollado una definición de caso preliminar (Tabla 1) y un formulario de informe de caso para el trastorno inflamatorio multisistémico en niños y adolescentes. La definición de caso preliminar refleja las características clínicas y de laboratorio observadas en niños informados hasta la fecha, y sirve para identificar 
Tabla 1: Definición preliminar del síndrome inflamatorio multisistémico en niños y adolescentes. ${ }^{10}$

Niños y adolescentes de 0 a 19 años con fiebre $>3$ días y dos de los siguientes:

- Erupción cutánea o conjuntivitis no purulenta bilateral o signos de inflamación mucocutánea (oral, manos o pies), hipotensión o choque, características de disfunción miocárdica, pericarditis, valvulitis o anomalías coronarias (incluidos hallazgos de ecocardiograma o troponina elevada/y la porción $\mathrm{N}$ terminal del propéptido natriurético tipo B [NT-pro-BNP]), evidencia de coagulopatía (PT, PTT y dímero D elevados), problemas gastrointestinales agudos (diarrea, vómitos o dolor abdominal)

- Marcadores elevados de inflamación, como VSG, proteína $C$ reactiva o procalcitonina y ninguna otra causa microbiana evidente de inflamación, incluida la sepsis bacteriana, los síndromes de choque estafilocócicos o estreptocócicos

- Evidencia de COVID-19 (RT-PCR, prueba de antígeno o serología positiva), o probable contacto con pacientes con COVID-19

PT = tiempo de protrombina (por sus siglas en inglés); PTT = tiempo de tromboplastina parcial (por sus siglas en inglés); VSG = velocidad de sedimentación globular; RT-PCR = reacción en cadena de la polimerasa con transcriptasa inversa (por sus siglas en inglés).

casos sospechosos o confirmados con el fin de proporcionar tratamiento e informes de vigilancia provisionales.

\section{LABORATORIO}

Las alteraciones en el laboratorio son menos comunes en los niños en comparación con los adultos.

Mientras que la linfopenia, la elevada proteína $C$ reactiva (PCR) y las pruebas de coagulación anormales son comunes en adultos y se correlacionan con gravedad de la enfermedad, no hay anormalidades de laboratorio consistentes en los estudios pediátricos. Sin embargo, se han reportado anormalidades de laboratorio que reflejan más de cerca los resultados de adultos en niños mayores de cinco años y en adolescentes. ${ }^{4,8-10}$

En grandes series de casos COVID-19 no parece aumentar los niveles de procalcitonina (PCT) y en general son $<0.05 \mathrm{mg} / \mathrm{mL}$ en $95 \%$ de los pacientes. En niños, la elevación de la PCT se ha evidenciado, pero se ha relacionado con coinfección, principalmente influenza. En adultos con COVID-19 se ha identificado además elevación del dímero $D$ y deshidrogenasa láctica. ${ }^{11}$

Los niveles de IL-1 e IL-6 se han utilizado para evaluar la respuesta hiperinflamatoria desencadena- da por SARS-CoV-2. Las experiencias en España e Italia nos han enseñado que podemos correlacionar de manera indirecta el aumento de ferritina, lactato deshidrogenasa (LDH), linfopenia y LDH con los niveles elevados de estas citocinas; esto ante la imposibilidad de medirlas en la mayoría de los laboratorios clínicos. Otro aprendizaje importante de Europa es la medición de los tiempos de coagulación, fibrinógeno, dímero $D$, al entender que la tormenta de citocinas tiene un gran compromiso a nivel de este sistema hematológico.

En el síndrome inflamatorio multisistémico en niños y adolescentes los reactantes de la fase aguda también están elevados, incluyendo la ferritina, pero se caracteriza por tener pruebas de función miocárdica alteradas, elevación de troponinas y del péptido natriurético cerebral, además de linfopenia marcada y trombocitopenia, similar a un síndrome de activación macrofágica.

\section{Reacción de la cadena de la polimerasa en tiempo real (RT-PCR)}

El principal método diagnóstico para SARS-CoV-2 es la RT-PCR de secreciones respiratorias altas y bajas. Existe variedad de genes ARN diana que pueden ser uno o más de la envoltura, nucleocápside, proteína Spike (S) y ARN polimerasa dependiente de ARN y genes ORF1. Es detectable desde el día uno de los síntomas y pico a la primera semana. La positividad disminuye a las tres semanas hasta hacerse indetectable.

Para COVID-19, los centros para el control y la prevención de enfermedades (CDC) recomiendan recolectar y analizar muestras de las vías respiratorias superiores (hisopado nasofaríngeo); también recomiendan analizar muestras de las vías respiratorias inferiores, si están disponibles.

Para los pacientes que desarrollan una tos productiva, el esputo se debe recolectar y analizar para detectar COVID-19. No se recomienda la inducción de esputo. Para los pacientes que están clínicamente indicados (por ejemplo aquellos que reciben ventilación mecánica invasiva) se debe recolectar un aspirado del tracto respiratorio inferior o una muestra de lavado broncoalveolar.

La sensibilidad de la RT-PCR es variable, para muestra de lavado broncoalveolar es de $93 \%$, esputo $72 \%$, nasofaríngeo $63 \%$ y faríngeo $32 \%$. También se ha reportado detección del virus en materia fecal, aunque su significancia clínica aún 
Rev Latin Infect Pediatr 2020; 33 (4): 165-173

es incierta. La especificidad de todas las muestras es de $100 \% .^{12}$

De acuerdo con el criterio de diagnóstico actual, la prueba de ácido nucleico viral mediante el ensayo de RT-PCR desempeña un papel vital en la determinación de la hospitalización y el aislamiento de pacientes individuales. Sin embargo, su falta de sensibilidad, estabilidad insuficiente y tiempo de procesamiento relativamente largo fueron perjudiciales para el control de la epidemia de la enfermedad. Además, varios factores externos pueden afectar los resultados de las pruebas de RT-PCR, incluidas las operaciones de muestreo, la fuente de muestras (tracto respiratorio superior o inferior) y el tiempo de muestreo (tiempo de la toma de muestra desde el inicio de los síntomas).

Como tal, los resultados de las pruebas de RT-PCR deben interpretarse con cautela. En casos muy sospechosos se debe tomar una segunda muestra del tracto respiratorio inferior si la primera es negativa y se realizó de nasofaringe. Además, la RT-PCR positiva no necesariamente indica presencia del virus viable. Algunos casos de sospecha de reinfección o reactivación podrían ser por errores del test y no han mostrado crecimiento del virus en cultivos celulares. Al ser difícil la interpretación de la PCR positiva posterior de la segunda semana, los CDC sugieren una «estrategia basada en síntomas» como requisito para que el personal de salud reingrese a laborar en los hospitales: al menos 72 horas sin fiebre y sin antipiréticos, resolución de síntomas respiratorios y que se esté en más del décimo día de enfermedad desde el primer día de síntomas. ${ }^{11}$

En el síndrome multisistémico de niños y adolescentes la RT-PCR en la mayoría de los casos es negativa, con IgG positiva en $80 \%$ de los pacientes, lo que hace sospechar que esta patología sea secundaria a un proceso de no regulación de la respuesta inmune y no a enfermedad aguda por SARS-CoV-2.

Los paneles respiratorios múltiples basados en reacción de cadena de polimerasa detectan los HCoV 229E, NL63, OC43 y HKU1, pero no funcionan para SARS-CoV-2, el cual requiere una RT-PCR específica. Sin embargo, una prueba positiva para $\mathrm{HCoV}$ u otro virus disminuye el índice de sospecha para COVID-19.

\section{Anticuerpos}

Aunque anticuerpos $\lg \mathrm{G}$ e $\lg \mathrm{M}$ han sido detectados desde el día cuatro, los niveles más altos están después de la segunda semana. La IgM desaparece entre la quinta y la séptima semana.

Los anticuerpos neutralizantes son contra la proteína de ataque del huésped: el dominio de unión al receptor de la proteína S. Sin embargo, las pruebas de anticuerpos producidas actúan contra proteínas de la nucleocápside. La sensibilidad de la IgM de la nucleocápside es baja (51.9\%). Existe un debate si estos anticuerpos tienen correlación con anticuerpos neutralizantes, al parecer según datos actuales los títulos elevados podrían indicar protección.

Existe preocupación por la interpretación de estos anticuerpos debido a la alta probabilidad de reacción cruzada con otros coronavirus (falsos positivos) y ante la escasa validación de estas pruebas que pueden llevar a tener falsos negativos; también desconocemos la duración y persistencia de la protección de anticuerpos neutralizantes en el tiempo. ${ }^{13}$

Como se mencionó anteriormente, el síndrome inflamatorio en niños y adolescentes se caracteriza por serologías positivas, lo que hace sospechar que la clínica sea secundaria a un proceso de infección por SARS-CoV-2 de dos a cuatro semanas previas a la aparición de los síntomas.

\section{HALLAZGOS RADIOLÓGICOS}

En niños el hallazgo en la radiografía de tórax más frecuente fue la opacidad bilateral en vidrio esmerilado $(32.7 \%)$. También se informan consolidados bilaterales a menudo en la periferia de los pulmones. En la tomografía axial computarizada (TAC) de tórax $32 \%$ de los pacientes tenían consolidaciones en vidrio esmerilado, $18.7 \%$ consolidados irregulares localizados, $12 \%$ consolidados irregulares bilaterales y $1.2 \%$ infiltrados intersticiales. ${ }^{9}$ Asimismo, se ha reportado en pediatría el signo del halo. La resolución de las lesiones en la TAC de tórax pueden ser tardías, incluso podrían verse cuando el niño esté asintomático y tenga una RT-PCR para COVID-19 de control negativa. ${ }^{14}$

\section{COINFECCIONES}

En tiempos en los que recibimos abundante información sobre COVID-19, es importante recordar que los menores de cinco años especialmente, presentan a menudo infecciones respiratorias y éstas se incrementan con la llegada del invierno. Existen pocos datos de coinfección viral en pacien- 
Rev Latin Infect Pediatr 2020; 33 (4): 165-173

tes positivos para SARS-CoV-2. Tres estudios, uno en China y dos en EUA variaron en sus resultados mostrando un porcentaje de coinfección de 2.99, 5 y $7.5 \%$, respectivamente. Rhinovirus/enterovirus, otros coronavirus humanos comunes, virus sincitial respiratorio, virus de Epstein Barr e influenza fueron los principales virus encontrados. En algunos reportes también se ha encontrado la coinfección de Mycoplasma en 20 a 26\%. ${ }^{13-15}$

Se ha propuesto que las bajas tasas de coinfección viral (especialmente con rhinovirus) pueda ser explicado porque algunos virus interfieren con la capacidad de establecer infección de otras especies virales. ${ }^{16}$

Además, a pesar de la baja proporción de coinfecciones, la presencia de un patógeno que no sea SARS-CoV-2 puede no proporcionar la seguridad de que un paciente tenga COVID-19, por lo menos hasta tener una RT-PCR detectada para el nuevo coronavirus. ${ }^{11,12}$

\section{TRATAMIENTO DE COVID-19}

Existen algunos reportes de las terapias farmacológicas que se han utilizado en pediatría, pero no es posible concluir que modifiquen favorablemente el curso clínico de la enfermedad. En adultos cabe mencionar el uso de lopinavir/ritonavir, remdesivir, interferón, ribavirina, glucocorticoides, inmunoglobulina (IGIV), inmunomoduladores y medicina tradicional china. Uno de los primeros medicamentos utilizados fue hidroxicloroquina, pero en los últimos estudios realizados ha llamado más su atención por sus efectos adversos, además de no tener efecto significativo en el curso clínico.

\section{Estudio solidaridad}

«Solidaridad» es un ensayo clínico aprobado por la OMS en adultos, con el objetivo de encontrar un tratamiento eficaz contra COVID-19. En el ensayo se compararán tres opciones de tratamiento con la norma asistencial para evaluar la eficacia relativa de cada una de ellas frente a COVID-19. Mediante la participación en el ensayo de pacientes en múltiples países, "Solidaridad» tiene por objeto descubrir con rapidez si alguno de los medicamentos estudiados retrasa la progresión de la enfermedad o mejora la tasa de supervivencia. Podrán incluirse más medicamentos en el ensayo en función de los datos que vayan apareciendo sobre ellos. ${ }^{17}$
Mientras no haya pruebas suficientes, la Organización Mundial de la Salud advierte que los médicos y las asociaciones médicas no deben administrar estos tratamientos no probados a pacientes con COVID-19 ni recomendarlos a personas que se automedican con ellos. La OMS está preocupada por informes sobre personas que se automedican con cloroquina y que se están ocasionando daños graves.

Los medicamentos incluidos en los estudios «Solidaridad» son: remdesivir, lopinavir/ritonavir y lopinavir/ritonavir + interferón beta-a. ${ }^{17}$

El 4 de julio de 2020, la OMS aceptó la recomendación del grupo directivo internacional del ensayo «Solidaridad» de interrumpir los grupos de tratamiento de COVID-19 con hidroxicloroquina y lopinavir/ritonavir.

Esta decisión se aplica a la continuación del ensayo "Solidaridad» en pacientes hospitalizados, pero no afecta a la posible evaluación de los efectos de la hidroxicloroquina y de la combinación lopinavir/ ritonavir en pacientes no hospitalizados como profilaxis previa o posterior a la exposición a COVID-19. Se están ultimando los resultados provisionales del ensayo "Solidaridad» con el fin de someterlos a una revisión científica externa y publicarlos. ${ }^{17}$

\section{Remdesivir}

Los investigadores de un ensayo clínico que comparó el antiviral remdesivir con placebo en pacientes con enfermedad pulmonar concluyeron que se había obtenido un beneficio en disminución de la mortalidad y días de hospitalización si el medicamento se inicia antes de la ventilación mecánica, lo que sugiere que este antiviral produce un mayor beneficio cuanto antes se inicie. Otro ensayo clínico aleatorizado (ECA), de China, no mostró ningún beneficio. ${ }^{18,19}$ Éste es un tratamiento experimental no autorizado ni disponible en la mayoría de los países de Latinoamérica.

\section{Lopinavir/ritonavir}

Las pocas publicaciones respecto al tratamiento de COVID-19 en pediatría indican el uso de lopinavir/ ritonavir, aunque su eficacia y seguridad en niños con COVID-19 es incierto. Este medicamento es un inhibidor de proteasa que inhibe la proteinasa principal del CoV, interrumpiendo su replicación. Sin embargo, en un ensayo clínico en pacientes hospitalizados con COVID-19, el uso de lopinavir/ritonavir 
Rev Latin Infect Pediatr 2020; 33 (4): 165-173

no demuestra utilidad en la mejoría clínica cuando es utilizado en monoterapia, pero en el análisis de intención a tratar modificado y en algunos desenlaces secundarios su uso fue favorable. ${ }^{20,21}$ Cabe señalar que el medicamento se administró relativamente tarde en el curso de la enfermedad en este estudio. Por lo que su uso debe ser considerado en el contexto de un experimento clínico aprobado y probablemente puede tener mayor impacto clínico en el paciente si se utiliza en los primeros días de la enfermedad.

\section{Inmunomoduladores}

Los resultados preliminares del ensayo "CONVACTA» que evalúa la eficacia y seguridad de tocilizumab en COVID-19 no alcanzó su objetivo primario: mejorar el estado clínico de pacientes adultos hospitalizados con neumonía grave asociada con COVID-19. Tampoco se ha detectado ningún problema de seguridad. Por el momento no se considera necesario adoptar ninguna medida regulatoria para suspender o paralizar temporalmente el reclutamiento de pacientes en ensayos clínicos. ${ }^{22}$ Se espera futuras investigaciones para definir su efectividad. Se encuentran en marcha estudios con otros inmunomoduladores como anakinra (IL-1) y están en desarrollo anticuerpos monoclonales específicos para SARS-CoV-2.

\section{Plasma convaleciente}

El plasma convaleciente o anticuerpos neutralizantes que contienen suero contra el SARS-CoV-2 se ha propuesto como un tratamiento útil. . $^{23,24}$ Sin embargo, un ECA realizado en China comparó el uso de plasma convaleciente más terapia estándar con solo la terapia estándar y no encontró diferencias significativas en la mortalidad a 28 días. La terapia estándar fue en su mayoría antivirales, antibacterianos, medicina tradicional China y en algunos pacientes se utilizó inmunoglobulina humana endovenosa (IGIV) e interferón. ${ }^{2}$

La Asociación Colombiana de Infectología (ACIN) en unión con el Instituto de Evaluación Tecnológica en Salud (IETS) han desarrollado un consenso nacional basado en el consenso de expertos e informado en la evidencia sobre atención, diagnóstico y manejo de la infección por SARS-CoV-2/ COVID-19, en el boletín 3, al tratar el punto del manejo de medicamentos actualiza en relación al uso de esteroides: ${ }^{27}$
- Se sugiere evitar el uso de corticosteroides de manera rutinaria en pacientes con infección por SARS-CoV-2.

- Se recomienda el uso de dexametasona en pacientes con requerimiento de oxígeno o ventilación mecánica, preferiblemente con más de siete días desde el inicio de síntomas. Se recomienda que la duración del tratamiento con corticoide sea hasta por 10 días.

- Para el uso de dexametasona se debe descartar infección por influenza; en caso de que ésta se documente, no se debe usar el medicamento.

- En caso de no haber disponibilidad de dexametasona se pueden utilizar dosis equivalentes de metilprednisolona, hidrocortisona o prednisolona.

- En pacientes que reciban esteroides es deseable el uso de ivermectina para profilaxis de infección por Strongyloides. En los casos en los que se encuentre contraindicado el uso de ivermectina por la edad del paciente se sugiere desparasitar con albendazol.

\section{AISLAMIENTO SOCIAL Y EL PAPEL DE LOS NIÑOS EN LA PANDEMIA}

Para reducir el $\mathrm{R} 0$ (índice reproductivo básico) o la tasa de infección se están utilizando políticas desde los gobiernos de cada país, entre las que se encuentran las restricciones de viajes, cuarentenas, ${ }^{28}$ y los niños en la mayoría de los países de Latinoamérica han dejado de ir a las instituciones educativas como método de aislamiento social.

Una de las principales preguntas que se hace la comunidad científica, los gobernantes y principalmente los padres es cuándo se reabrirá la educación presencial de los niños; sin embargo, son pocos los datos para responder esta interrogante que aún sigue sin respuesta.

Las hipótesis iniciales indicaban que el aislamiento de la población infantil era importante por la capacidad de los niños de ser mayores transmisores del virus que los adultos. Sin embargo, los argumentos para la transmisión del SARS-CoV-2 en las escuelas no son convincentes, al contrario del virus de la influenza.

Los efectos adversos del cierre de las escuelas pueden incluir la falta de educación e interacción social, afectando desproporcionadamente a los niños más vulnerables y desfavorecidos. ${ }^{29}$ Por lo 
tanto, se han sugerido otras medidas además de cerrar las escuelas para disminuir la transmisión de COVID-19, como promover la higiene y el distanciamiento físico en las escuelas. ${ }^{30,31}$ Un estudio realizado en Australia apoya esta propuesta. Se describen nueve estudiantes y nueve empleados con COVID-19 de 15 escuelas que tuvieron contacto cercano con 735 estudiantes y 128 empleados. Sólo dos infecciones secundarias se identificaron, ninguna en adultos, un estudiante fue potencialmente infectado por un miembro del personal y otro estudiante tuvo contacto con dos compañeros de escuela infectados. ${ }^{32}$

Además, existen otros estudios que indican que en los hogares los niños representan un bajo porcentaje de casos índice (aproximadamente 8\% en 39 hogares), y los niños desarrollaron síntomas después de los adultos. Otros estudios publicados también sugieren que el niño no era la fuente de infección y que la mayoría adquirieron COVID-19 de adultos en lugar de transmitirlo a ellos. ${ }^{33-36}$

Otro punto importante es que las cargas virales de SARS-CoV-2 fueron similares a las de otros grupos de edad, lo que aumenta la preocupación de que los niños podrían ser tan infecciosos como los adultos, ${ }^{37}$ pero al ser levemente sintomáticos podrían liberar menos partículas infecciosas al ambiente. ${ }^{34}$

En conclusión, es conocido que el cierre de las instituciones educativas puede aumentar las desigualdades sociales en los países latinoamericanos, ya que muchos niños no tienen la tecnología para desarrollar la modalidad de educación virtual utilizada en el confinamiento. Por lo tanto, los gobiernos deben considerar, en tiempos de COVID-19, la manera de incorporar la presencialidad escolar gradualmente, cabe señalar que se podrían evaluar modelos ya instaurados en otros países, pero teniendo en cuenta que el aislamiento social y la higiene de manos continúa siendo la manera más eficaz de controlar la expansión del virus, al no tener a disposición una vacuna o tratamiento efectivo a corto plazo.

\section{PROFILAXIS Y VACUNAS}

Se están realizando estudios para desarrollar una vacuna efectiva. Se desconoce si la infección al igual que la futura vacuna confieren inmunidad parcial o completa y la duración de esa protección, además de la interpretación de las pruebas serológicas para evaluar la efectividad de la vacuna. ${ }^{38}$

\section{REFERENCIAS}

1. Wu Z, McGoogan JM. Characteristics of and important lessons from the coronavirus disease 2019 (COVID-19) outbreak in China: summary of a report of 72314 cases from the Chinese Center for Disease Control and Prevention. JAMA. 2020; 323 (13): 1239-1242.

2. Dong $Y, M o X, H u$ Y et al. Epidemiological characteristics of 2143 pediatric patients with 2019 coronavirus disease in China. Pediatrics. 2020. doi: 10.1542/peds.2020-0702.

3. Liu W, Zhang Q, Chen J, Xiang R, Song H, Shu S et al. Detection of Covid-19 in children in early january 2020 in Wuhan, China. N Engl J Med. 2020; 382 (14): 1370-1371.

4. Singh $\mathrm{T}$, Heston $\mathrm{SM}$, Langel $\mathrm{SN}$, Blasi M, Hurst JH, Fouda GG et al. Lessons from COVID-19 in children: key hypotheses to guide preventative and therapeutic strategies. Clin Infect Dis [Internet]. 2020; ciaa547. Available in: https:// doi.org/10.1093/cid/ciaa547

5. Riphagen S, Gomez X, Gonzalez-Martinez C, Wilkinson N, Theocharis P. Hyperinflammatory shock in children during COVID-19 pandemic. Lancet. 2020; 395 (10237): 16071608.

6. Hamiel U, Kozer E, Youngster I. SARS-CoV-2 rates in BCGvaccinated and unvaccinated young adults. JAMA. 2020; 323 (22): 2340-2341. doi: 10.1001/jama.2020.8189.

7. Fang F, Luo XP. Facing the pandemic of 2019 novel coronavirus infections: the pediatric perspectives. Zhonghua Er Ke Za Zhi. 2020; 58 (0): E001.

8. Garazzino S, Montagnani $C$, Donà D, Meini A, Felici E, Vergine $\mathrm{G}$ et al. Multicentre Italian study of SARS-CoV-2 infection in children and adolescents, preliminary data as at 10 April 2020. Euro Surveill. 2020; 25 (18): 2000600. doi: 10.2807/1560-7917.ES.2020.25.18.2000600.

9. Shekerdemian LS, Mahmood NR, Wolfe KK, Riggs BJ, Ross $\mathrm{CE}$, McKiernan CA et al. Characteristics and outcomes of children with coronavirus disease 2019 (COVID-19) infection admitted to US and Canadian Pediatric Intensive Care Units. JAMA Pediatr. 2020. doi: 10.1001/ jamapediatrics.2020.1948.

10. World Health Organization. Multisystem inflammatory syndrome in children and adolescents with COVID-19. 2020. WHO reference number: WHO/2019-nCoV/Sci_Brief/ Multisystem_Syndrome_Children/2020.1

11. Nowak MD, Sordillo $\bar{E} \bar{M}$, Gitman MR, Paniz Mondolfi AE. Co-infection in SARS-CoV-2 infected patients: where are influenza virus and rhinovirus/enterovirus? J Med Virol. 2020: 10.1002/jmv.25953.

12. Kim D, Quinn J, Pinsky B, Shah NH, Brown I. Rates of co-infection between SARS-CoV-2 and other respiratory pathogens. JAMA. 2020; 323 (20): 2085-2086.

13. Sethuraman $N$, Jeremiah SS, Ryo A. Interpreting diagnostic tests for SARS-CoV-2. JAMA. 2020; 323 (22): 2249-2251.

14. Xia W, Shao J, Guo Y, Peng X, Li Z, Hu D. Clinical and CT features in pediatric patients with COVID-19 infection: Different points from adults. Pediatr Pulmonol. 2020; 55 (5): 1169-117.

15. Zimmermann P, Curtis N. COVID-19 in children, pregnancy and neonates: a review of epidemiologic and clinical features. Pediatr Infect Dis J. 2020; 39 (6): 469-477.

16. Lopez-Medina E, Ardura MI, Siegel JD, Brock E, Sánchez PJ. 2009 influenza $A$ in infants hospitalized at younger than 6 months. J Pediatr. 2012; 160 (4): 626-631.e1.

17. OMS. Ensayo clínico "Solidaridad" sobre tratamientos contra la COVID-19. 2020. Disponible en: https://www. 
Rev Latin Infect Pediatr 2020; 33 (4): 165-173

who.int/es/emergencies/diseases/novel-coronavirus-2019/ global-research-on-novel-coronavirus-2019-ncov/solidarityclinical-trial-for-covid-19-treatments

18. Beigel JH, Tomashek KM, Dodd LE, Mehta AK, Zingman BS, Kalil AC et al. Remdesivir for the treatment of COVID-19 - Preliminary Report. N Engl J Med. 2020: NEJMoa2007764.

19. Wang Y, Zhang D, Du G, Du R, Zhao J, Jin Y et al. Remdesivir in adults with severe COVID-19: a randomised, double-blind, placebo-controlled, multicentre trial. Lancet. 2020; 395 (10236): 1569-1578.

20. Cao B, Wang Y, Wen D, Liu W, Wang J, Fan G et al. A trial of lopinavir-ritonavir in adults hospitalized with severe Covid-19. N Engl J Med. 2020; 382 (19): 1787-1799.

21. Bhimraj A, Morgan RL, Shumaker AH, Lavergne V, Baden $\mathrm{L}$, Cheng VC et al. Infectious diseases Society of America Guidelines on the treatment and management of patients with COVID-19. Clin Infect Dis. 2020: ciaa478.

22. Anonymous Roche provides an update on the phase III COVACTA trial of Actemra/RoActemra in hospitalised patients with severe COVID-19 associated pneumonia. Available in: https://www.roche.com/investors/updates/invupdate-2020-07-29.htm

23. Cheng $Y$, Wong R, Soo YO et al. Use of convalescent plasma therapy in SARS patients in Hong Kong. Eur J Clin Microbiol Infect Dis. 2005; 24 (1): 44-46.

24. Shen C, Wang Z, Zhao F, Yang Y, Li J, Yuan J et al. Treatment of 5 critically ill patients with COVID-19 with convalescent plasma. JAMA. 2020; 323 (16): 1582-1589.

25. Li L, Zhang W, Hu Y, Tong X, Zheng S, Yang J et al. Effect of convalescent plasma therapy on time to clinical improvement in patients with severe and life-threatening COVID-19: a randomized clinical trial. JAMA. 2020; 324 (5): 460-470.

26. Ranieri VM, Pettilä V, Karvonen MK, Jalkanen J, Nightingale $P$, Brealey $D$ et al. Effect of intravenous interferon $\beta-1$ a on death and days free from mechanical ventilation among patients with moderate to severe acute respiratory distress syndrome: a randomized clinical trial. JAMA. 2020.

27. Saavedra Trujillo CH. Consenso colombiano de atención, diagnóstico y manejo de la infección por SARS-CoV-2/ COVID 19 en establecimientos de atención de la salud. Recomendaciones basadas en consenso de expertos e informadas en la evidencia, Infectio. 2020; 24 (3): 1-153.
28. Chinazzi M, Davis JT, Ajelli M, Gioannini C, Litvinova $M$, Merler $S$ et al. The effect of travel restrictions on the spread of the 2019 novel coronavirus (COVID-19) outbreak. Science. 2020; 368 (6489): 395-400.

29. Cauchemez S, Valleron AJ, Boelle PY, Flahault A, Ferguson NM. Estimating the impact of school closure on influenza transmission from Sentinel data. Nature. 2008; 452 (7188): 750-754. doi: 10.1038/nature06732.12.

30. Litvinova M, Liu QH, Kulikov ES, Ajelli M. Reactive school closure weakens the network of social interactions and reduces the spread of influenza. Proc Natl Acad Sci U S A. 2019; 116 (27): 13174-13181. doi: 10.1073/ pnas. 1821298116 .

31. Armitage R, Nellums LB. Considering inequalities in the school closure response to COVID-19. Lancet Glob Health. 2020; 8 (5): e644. doi: 10.1016/S2214-109X(20)30116-9.

32. COVID-19 in schools-the experience in NSW. National Centre for Immunisation Research and Surveillance. New South Wales, Australia. April 26, 2020.

33. Posfay-Barbe KM, Wagner N, Gauthey M, Moussaoui D, Loevy N, Diana A et al. COVID-19 in children and the dynamics of infection in families. Pediatrics. 2020; 146 (2): e20201576.

34. Lee B, Raszka WV Jr. COVID-19 transmission and children: the child is not to blame. Pediatrics. 2020; 146 (2): e2020004879.

35. Wu Q, Xing Y, Shi L, Li W, Gao Y, Pan S et al. Coinfection and other clinical characteristics of COVID-19 in children. Pediatrics. 2020; 146 (1): e20200961.

36. Jiehao C, Jin X, Daojiong L, Zhi Y, Lei X, Zhenghai Q et al. A case series of children with 2019 novel coronavirus infection: clinical and epidemiological features. Clin Infect Dis. 2020; 71 (6): 1547-1551.

37. Jones TC, Jones TC, Mühlemann B, Veith T, Biele G, Zuchowski M, Hoffmann J et al. An analysis of SARS-CoV-2 viral load by patient age. medRxiv. 2020 .

38. Mayo Clinic. Vacuna contra la COVID-19 (coronavirus): obtén la información verdadera. 2020. Disponible en: www. mayoclinic.org/es-es/coronavirus-vaccine/art-20484859

Correspondencia:

Dr. Iván Benavides Reina

E-mail:medicoputumayo@hotmail.com 